\title{
Comportamiento magnético del acero AISI 304 en ensayos de tracción uniaxial
}

\section{Magnetic behavior of AISI 304 steel in uniaxial tensile tests}

\author{
Miriam Rocío Neyra Astudillo ${ }^{1,2,3}$, Hugo Juan Scagnetti ${ }^{4}$, Nicolás Nuñez ${ }^{1}$, \\ Guido Ferrari $^{1,2}$, Maria Isabel López Pumarega ${ }^{1}$, Martin Gomez ${ }^{1,2}$, \\ José Ruzzante ${ }^{2,4,5}$, Linilson Padovese Rodrigues ${ }^{6}$
}

\footnotetext{
${ }^{1}$ Dpto. Proyecto ICES y Ondas Elásticas, Comisión Nacional de Energía Atómica CNEA, Av. G. Paz 1499, San Martín, Buenos Aires, Argentina

${ }^{2}$ Universidad Tecnológica Nacional UTN, Fac. Reg. Delta, Buenos Aires, Argentina.

${ }^{3}$ IT Sabato, UNSAM-CNEA, Av. G. Paz 1499, San Martin, Buenos Aires, Argentina.

${ }^{4}$ Universidad Nacional de Tres de Febrero, UNTREF, Caseros, Buenos Aires, Argentina.

${ }^{5}$ Universidad Nacional de Chilecito, UNdeC, Chilecito, La Rioja, Argentina

${ }^{6}$ Departamento de Engenharia Mecânica, Escola Politécnica, Universidade de São Paulo, São Paulo, Brasil. e-mail: neyra@cnea.gov.ar, hugoscagnetti@hotmail.com,nnunez@cnea.gov.ar, guicferrari@gmail.com, lopezpum@cnea.gov.ar,mpgomez@cnea.gov.ar,ruzzante@gmail.com,1rpadove@usp.br
}

\section{RESUMEN}

Durante el proceso de magnetización de un material ferromagnético, las paredes de los dominios magnéticos son forzadas a moverse bajo la influencia del campo magnético. Para que las paredes se puedan mover deben superar la resistencia producida por los puntos de anclaje (borde de grano, dislocaciones, inclusiones, etc.), ocasionando saltos discretos en la magnetización. Estos saltos se pueden detectar con una bobina sensora colocada sobre la superficie de la muestra, que los transforma en pulsos de voltaje. Estos últimos son conocidos como Ruido Magnético Barkhausen (RMB).

En este trabajo se continúa el estudio de ensayos de tracción uniaxial, hasta rotura, en probetas de acero AISI 304 (no magnético), en las cuales, como consecuencia de la deformación, aparece una fase ferromagnética que sí produce RMB. Las mediciones se realizaron a diferentes cargas con el fin de observar cómo cambiaban las características del RMB. A partir de las señales obtenidas en las mediciones, se diseñó un filtro digital con el propósito de eliminar todo tipo de señal espuria e individualizar el RMB. Para tal propósito se utilizó un filtro digital Butterworth con banda pasante (2- 200) $\mathrm{kHz}$. La selección del tipo de filtro se debe a que éste presenta una respuesta prácticamente constante hasta la frecuencia de corte, obteniéndose una respuesta plana a lo largo de la banda pasante. Para cada valor de carga se analizaron por separado las señales de RMB respecto de las etapas de aumento y disminución del campo magnético de excitación. Con el objetivo de caracterizar cada etapa, se estimaron diversos parámetros estadísticos que fueron evaluados en función de la deformación aplicada. Por último se calculó la evolución temporal de las señales y su función cuadrática media (RMS) para cada etapa de la magnetización, realizándose la correlación cruzada con el propósito de evaluar el grado de semejanza existente entre cada parte de la señal.

Palabras clave: Ruido magnético Barkhausen, austenítico, martensítica, ensayos de deformación.

\section{ABSTRACT}

During the magnetization process of a ferromagnetic material, the magnetic domain walls are forced to move due to the magnetic field influence. The domain walls must overcome the stress produced by the pinning sites (grain boundary, dislocations, inclusions, etc.), producing discrete jumps in the magnetization. These jumps can be detected with a sensor coil located on the surface sample, transforming them in voltage pulses known as Magnetic Barkhausen Noise (MBN).

This work is the follow-up of the study on the uniaxial tensile test up to breakage, on AISI 304 steel (nonmagnetic) samples. As a consequence of the strain a new ferromagnetic phase appears which produces MBN. From the signals obtained during the measurements, a digital filter was designed and applied in order to elim- 
inate any spurious signal and to identify the MBN. For this purpose a Butterworth digital filter was used with a (2-200) kHz bandwidth. This filter was selected because it presents a practically constant response up to the cutoff frequency, giving a flat response over the entire passband. For each load value, the MBN signals were analyzed separately concerning the stages of increase and decrease of the magnetic field excitation. In order to characterize each stage, various statistical parameters were evaluated as a function of the applied strain. Finally, the time evolution of the signals and their root mean square function (RMS) were calculated for each stage of the magnetization, calculating the cross correlation function.

Keywords: Magnetic Barkhausen noise, austenitic steel, martensitic transformation, uniaxial tensile tests.

\section{INTRODUCCIÓN}

El acero inoxidable austenítico 304, que es metaestable a temperatura ambiente, se transforma en fase martensita, ya sea por enfriamiento a temperatura bajo cero o por deformación plástica. Esta nueva fase, en el último caso, se denomina martensita inducida por deformación. Existen dos tipos de martensita inducida, martensita $\varepsilon$ con una estructura cristalina hcp y la martensita $\alpha^{\prime}$ con una estructura cristalina bcc. Durante la deformación, la martensita $\alpha^{\prime}$ puede ser producida a partir de la fase de austenita y/o directamente de la martensita $\varepsilon$. La magnitud de la transformación depende del tamaño de grano, la composición química, la temperatura y el porcentaje de deformación. Por otro lado, es sabido que la deformación plástica en los materiales policristalinos genera también una textura cristalográfica particular [1-5].

El Ruido Magnético Barkhausen (RMB) es una técnica de ensayo no destructivo y es ampliamente utilizado para la caracterización microestructural de materiales ferromagnéticos. Esta técnica se basa principalmente en los movimientos irreversibles de las paredes de los dominios magnéticos que deben superar los puntos de anclaje, como bordes de grano, dislocaciones, precipitados, etc.. Estas imperfecciones impiden el movimiento de las paredes de los dominios hasta que un aumento en el campo magnético aplicado, proporciona la energía necesaria para liberar a las paredes de dichos obstáculos. Estos puntos de anclaje producen un salto repentino en la magnetización produciendo un cambio de flujo llamado RMB [6-9].

En la presente investigación, se usa la técnica de RMB para estudiar la fase magnética inducida por deformación en el acero AISI 304.También se presenta la caracterización metalográfica de dicho acero. Este material ha sido elegido para este estudio, ya que transforma la fase austenítica (no magnética), en la fase martensítica (ferromagnética), durante la deformación a temperatura ambiente, permitiendo la adecuada implementación del ensayo monitorizado con esta técnica.

Previamente se ensayaron 8 probetas mediante tracción uniaxial hasta la rotura, registrándose las señales de RMB a diferentes valores de carga [8,9]. En este trabajo, a partir de las señales obtenidas en las mediciones, se diseñó un filtro en Matlab con el propósito de disminuir todo tipo de ruido e individualizar más claramente el RMB. Para tal propósito se utilizó un filtro digital Butterworth con banda pasante hasta 200 $\mathrm{kHz}$. La selección del tipo de filtro se debe a que éste presenta una respuesta prácticamente constante hasta la frecuencia de corte, obteniéndose una respuesta plana a lo largo de la banda pasante. Para cada valor de carga se analizaron por separado las señales de RMB respecto al aumento y la disminución del campo magnético de excitación. Con el objetivo de caracterizar cada etapa de la magnetización, se estimaron diversos parámetros estadísticos que fueron evaluados en función de la deformación aplicada. Por último se calculó la evolución temporal del valor cuadrático medio (RMS) para cada etapa de la magnetización, realizándose la correlación cruzada entre cada uno de ellos para su comparación.

\section{MATERIALES Y MÉTODOS}

En esta sección se describen los materiales, procedimientos y métodos utilizados en la parte experimental del trabajo.

\subsection{Material}

El material utilizado es un acero inoxidable austenítico AISI 304, fabricado por el Grupo Arcelor Mittal Inox de Brasil y entregado en forma de placas ( $300 \mathrm{~mm} \times 210 \mathrm{~mm}$ x 0,5 $\mathrm{mm}$ ), laminadas en frio. Se prepararon 8 probetas para ensayos de tracción uniaxial bajo la Norma ASTM E8, 4 cortadas en la dirección de laminación $\left(0^{\circ}\right)$ y 4 cortadas en la dirección perpendicular $\left(90^{\circ}\right)$. En la Figura 1 se muestra una fotografía de la probeta, indicando sus dimensiones específicas. Una probeta de cada tipo $\left(0^{\circ}\right.$ y $\left.90^{\circ}\right)$ fue ensayada hasta rotura para encontrar sus propiedades mecánicas [8]. 


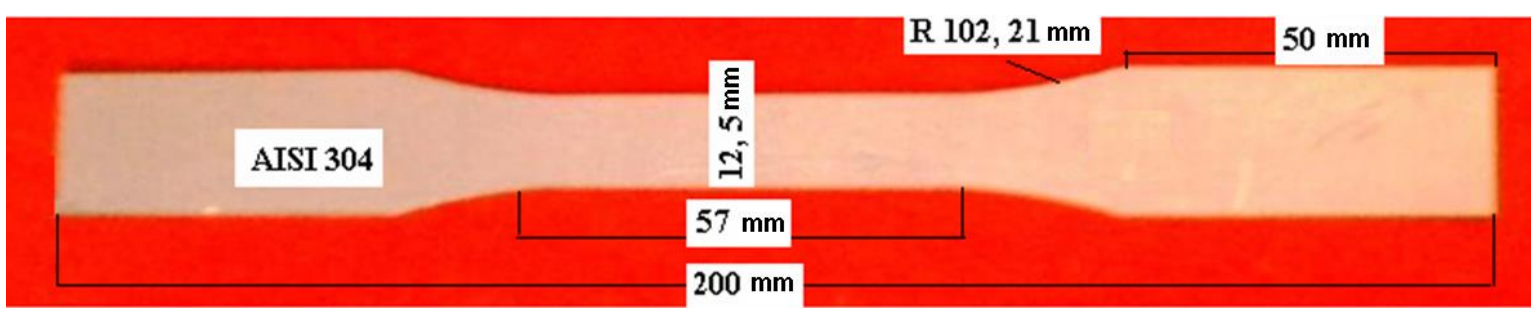

Figura 1: Dimensiones de las probetas según la Norma ASTM E 8.

El análisis EDAX realizado confirmó que el material correspondía al acero inoxidable austenítico AISI 304. Se midió la microdureza Vickers con una carga de $0.1 \mathrm{~kg}$. Se evaluó el porcentaje de martensita total usando un ferrideltímetro directamente sobre la muestra. Las dos mediciones se realizaron en la posición de máxima deformación, es decir cerca de la fractura [8-10].

Para el estudio de la microestructura se hizo un análisis con microscopia óptica y electrónica de barrido. Todas las probetas fueron atacadas electrolíticamente con ácido oxálico al $10 \%$ con una tensión de 10 V durante un $1 \mathrm{~min}$. Para poder observarlas con el microscopio electrónico de barrido se tuvo que sobre atacar las muestras por un minuto más. En la Tabla 1 se indica, para cada probeta, la deformación final alcanzada, la velocidad media de carga, el porcentaje total de martensita y la microdureza Vickers [8].

Tabla 1: Valores de velocidad de carga media, porcentaje de deformación final, de martensita total y microdureza Vickers para cada probeta.

\begin{tabular}{l|l|l|l|l}
\hline PROBETA & $\begin{array}{c}\text { Deformación } \\
\text { final } \\
(\boldsymbol{\varepsilon} \%)\end{array}$ & $\begin{array}{c}\text { Velocidad de } \\
\text { carga media } \\
\mathbf{( N / s )}\end{array}$ & $\begin{array}{c}\text { Martensita } \\
\text { total } \\
(\mathbf{M} \%)\end{array}$ & $\begin{array}{c}\text { Microdureza } \\
\text { Vickers } \\
\left(\mathbf{H V}_{\mathbf{0 , 1}}\right)\end{array}$ \\
\hline $0^{\circ}-1$ & 0 & 0 & $0,5 \pm 0,05$ & $192 \pm 6$ \\
\hline $0^{\circ}-2$ & $36 \pm 2,2$ & $22 \pm 2$ & $26 \pm 2,6$ & $401 \pm 12$ \\
\hline 0 -3 & $60 \pm 3,6$ & $27 \pm 2$ & $37 \pm 3,7$ & $432 \pm 13$ \\
\hline $90^{\circ}-1$ & $52 \pm 3,2$ & $20 \pm 2$ & $51 \pm 5,1$ & $467 \pm 14$ \\
\hline $90^{\circ}-2$ & $56 \pm 3,4$ & $15 \pm 2$ & $49 \pm 4,9$ & $456 \pm 14$ \\
\hline $90^{\circ}-3$ & $54 \pm 3,2$ & $10 \pm 2$ & $52 \pm 5,2$ & $430 \pm 13$ \\
\hline
\end{tabular}

Todos los ensayos se llevaron a cabo a temperatura ambiente con diferente velocidad media de deformación, debido a que se usó una máquina de tracción hidráulica y no fue posible lograr una velocidad constante. La probeta $0^{\circ}-2$ sólo se ensayó hasta el $36 \%$ de deformación, ya que luego se produjo su deslizamiento del soporte adicional que permitía mantener la probeta dentro del solenoide que producía la excitación magnética.

\subsection{Medición del RMB}

Los ensayos se realizaron en una máquina de tracción hidráulica AMSLER 60ZD 1638. Para producir el RMB se colocó la probeta dentro de un solenoide que la excitaba con un campo magnético variable. Este campo se generó con una corriente sinusoidal de $10 \mathrm{~Hz}$ proveniente de un amplificador excitado por un generador de función LeCroy ARBStudio 1102. El valor máximo de campo magnético de excitación fue de 0,553 $\mathrm{kA} / \mathrm{m}$. El RMB se midió con un sensor (bobina colectora) fijado en la parte central de la probeta. La amplitud máxima de la corriente de excitación en el solenoide fue de 0,075 A, medida con una resistencia en serie de $10 \mathrm{Ohm}$. El sensor de RMB se conectó a un amplificador con un ancho de banda de (2-200) kHz, con bajo nivel de ruido. Mientras la probeta se iba deformando por el aumento de la carga, se registró el RMB hasta la ruptura final. Esto se realizó adquiriendo las señales con un osciloscopio PicoScope 4227 controlado por una PC. Para cada ensayo, se emplearon 2 canales: uno para la tensión de excitación, y el otro para el RMB. Todas las señales, aproximadamente 20 por cada probeta, se digitalizaron con una frecuencia de 2,4 MSamples/s. En la Figura 2 se muestra un esquema del sistema de medición [8,11]. 


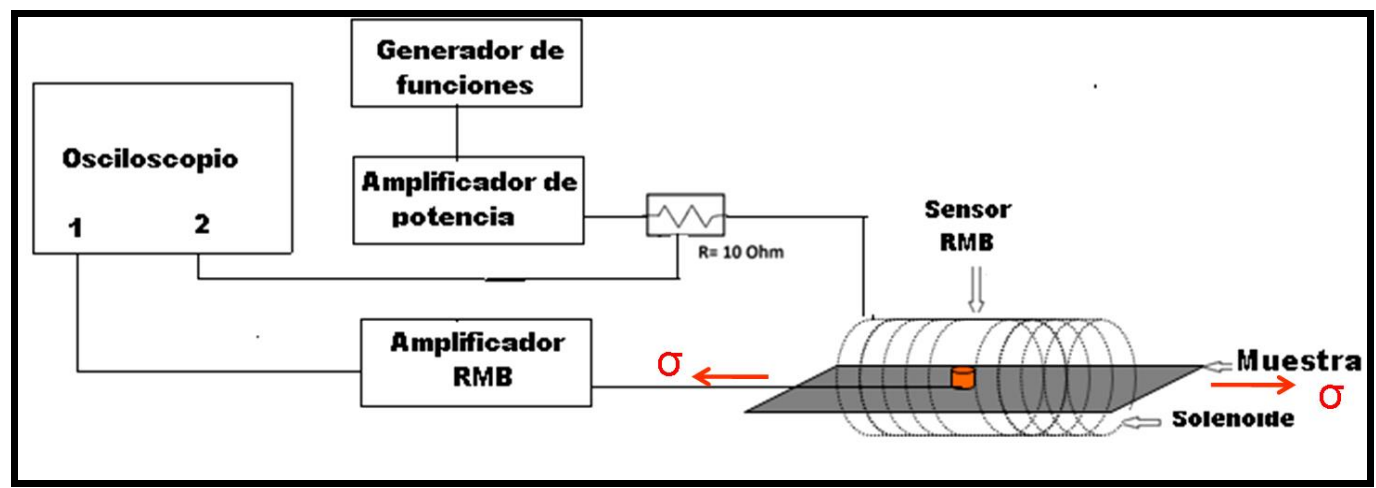

Figura 2: Esquema del sistema de medición del RMB.

\section{RESULTADOS Y DISCUSION}

En las siguientes dos secciones se detallan los resultados del análisis microestructural y las mediciones magnéticas.

\subsection{Análisis microestructural}

Se realizó un análisis de espectroscopía de energía dispersiva (EDS) que corroboró la presencia fundamental de Fe, Cr y Ni.

En las Figuras 3 a 8, se muestran las micrografías ópticas y electrónicas de barrido para las probetas ensayadas con diferente porcentaje de deformación final $(\varepsilon)$. En cada caso se indica el nombre de la probeta. Además se indica el porcentaje de martensita (M). En 3 a) se observa, para una probeta sin deformar, una estructura austenítica con granos equiaxiales con una variación entre $10 \mu \mathrm{m}$ y $50 \mu \mathrm{m}$ con una leve precipitación de carburos y maclas de recocido. Esto fue corroborado por la microscopía electrónica de barrido en b). A pesar de ser una probeta sin deformación axial, se midió un $0.5 \%$ de martensita probablemente producida durante el proceso de la laminación de la placa.
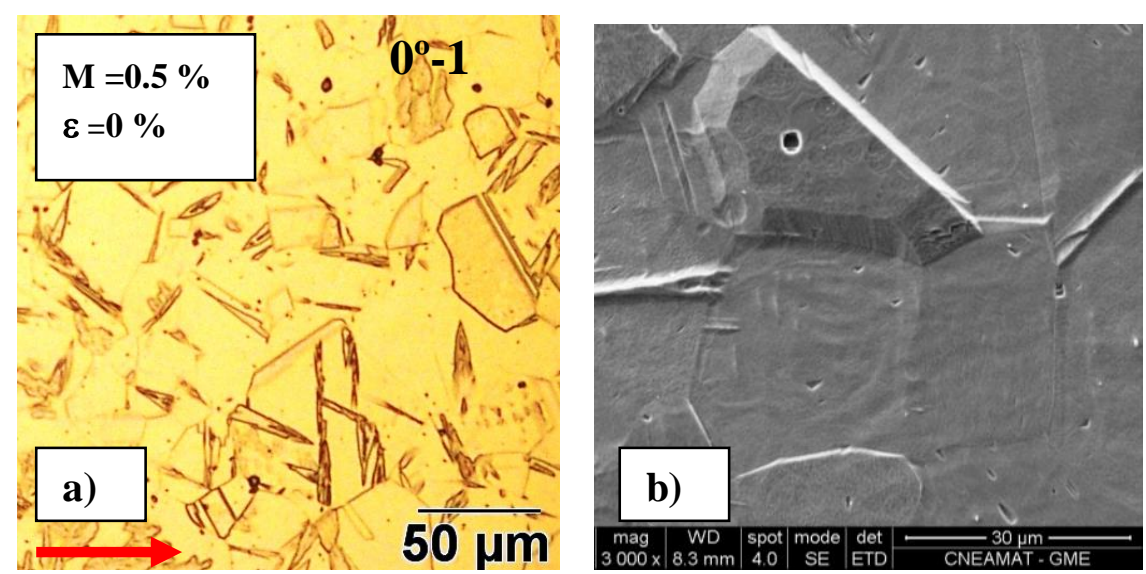

Figura 3: Fotografías a) microscopia óptica y b) microscopia electrónica de barrido (3000 x), para la probeta sin deformación, la flecha roja indica el sentido de laminación.

Las Figuras 4 y 5 corresponden a las probetas cortadas y deformadas en el sentido de laminación $\left(0^{\circ}\right)$ para el $26 \%$ y $37 \%$ de martensita. En el análisis de microscopía óptica (4 a)), se observan agujas finas de martensita distribuidas al azar en la matriz austenítica, lo que se corrobora también con la microscopía electrónica de barrido en 4 b); en 4 c) se muestra una amplificación a 8000 x. En 5 a) con microscopia óptica se ven agujas finas de martensitas distribuidas con una orientación más definida. En 5 b) la microscopia electrónica muestra que algunos granos presentan doble orientación de las agujas de martensita (figura 5 c)). 

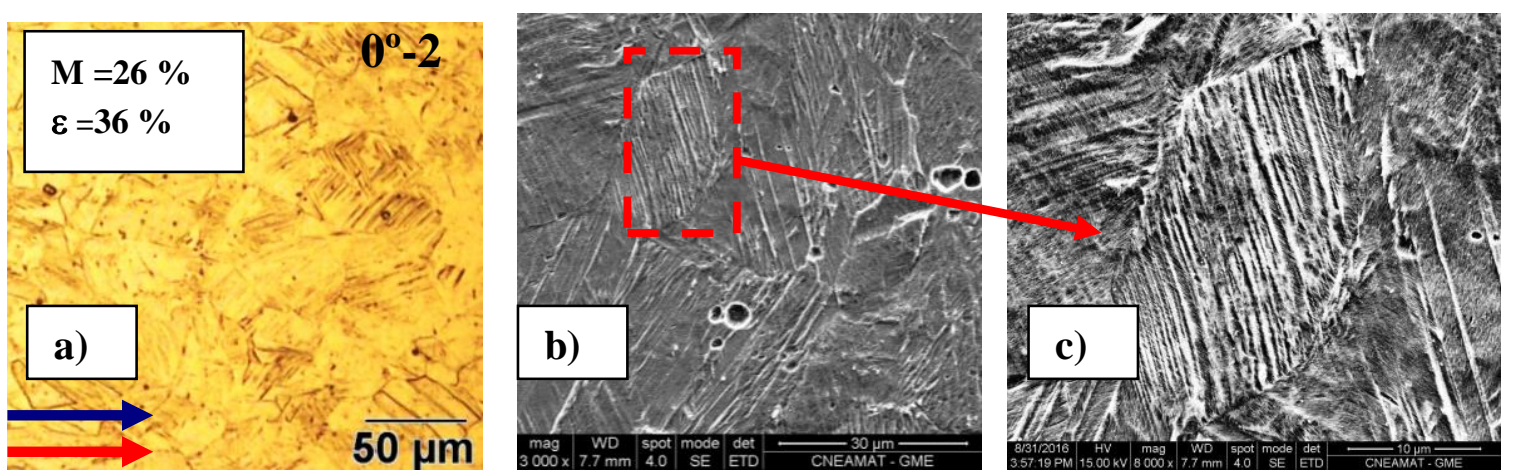

Figura 4: Fotografías a) microscopia óptica, b) microscopia electrónica de barrido (3000x), y c) amplificación 8000 x, para la probeta $0^{\circ}-2$. La fecha roja indica el sentido de laminación y la fecha azul indica el sentido de la deformación.
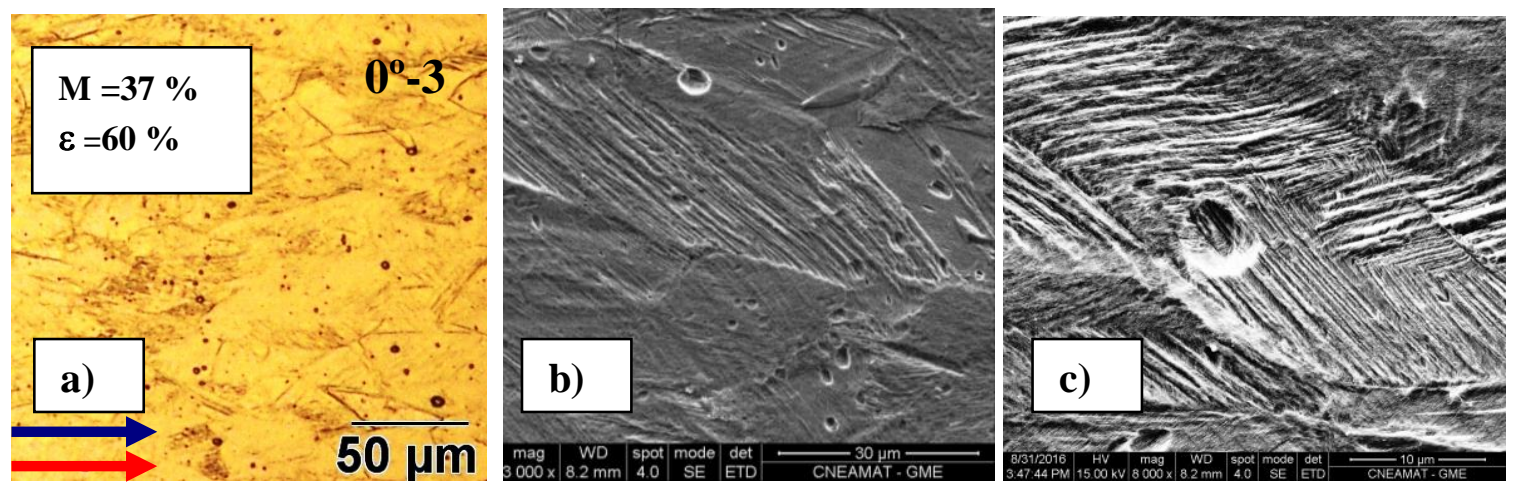

Figura 5: Fotografías a) microscopia óptica, b) microscopia electrónica de barrido (3000x), y c) amplificación 8000 x, para la probeta $0^{\circ}-3$. La fecha roja indica el sentido de laminación y la fecha azul indica el sentido de la deformación.

Las Figuras 6, 7 y 8 corresponden a la deformación de las probetas cortadas perpendicularmente al sentido de laminación $\left(90^{\circ}\right)$, para $51 \%, 49 \%$ y $52 \%$ de martensita. Como no hay mucha diferencia en el porcentaje de deformación final, se analizan todas ellas en conjunto. Con microscopia óptica, se observa una dirección preferencial en la formación de martensita, coincidente con la deformación, también una disminución de las distancias entre las interfaces austenita-martensita, ya que hay gran cantidad de martensita. En el análisis del microscopio electrónico de barrido se observan formaciones de agujas con simple y doble orientación dentro del mismo grano, además formación de maclas e inclusiones identificadas posteriormente como Al.

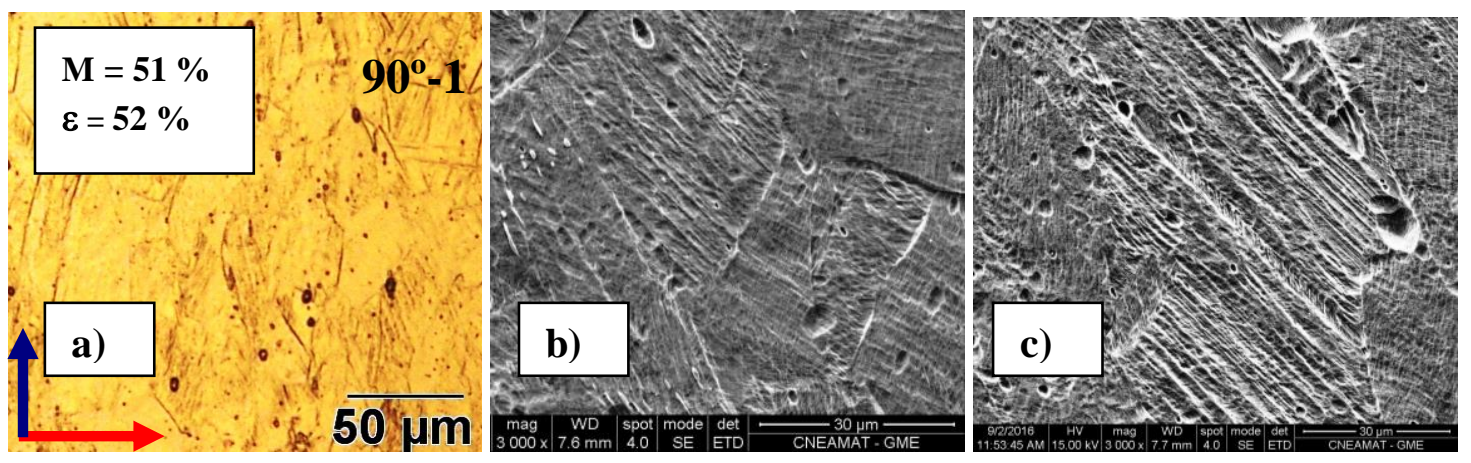

Figura 6: Imágenes: a) microscopia óptica, b) microscopia electrónica de barrido (3000 x) y c) otra zona con amplificación 3000 x, probeta $90^{\circ}$-1. La flecha roja indica el sentido de laminación y la azul indica el sentido de la deformación. 

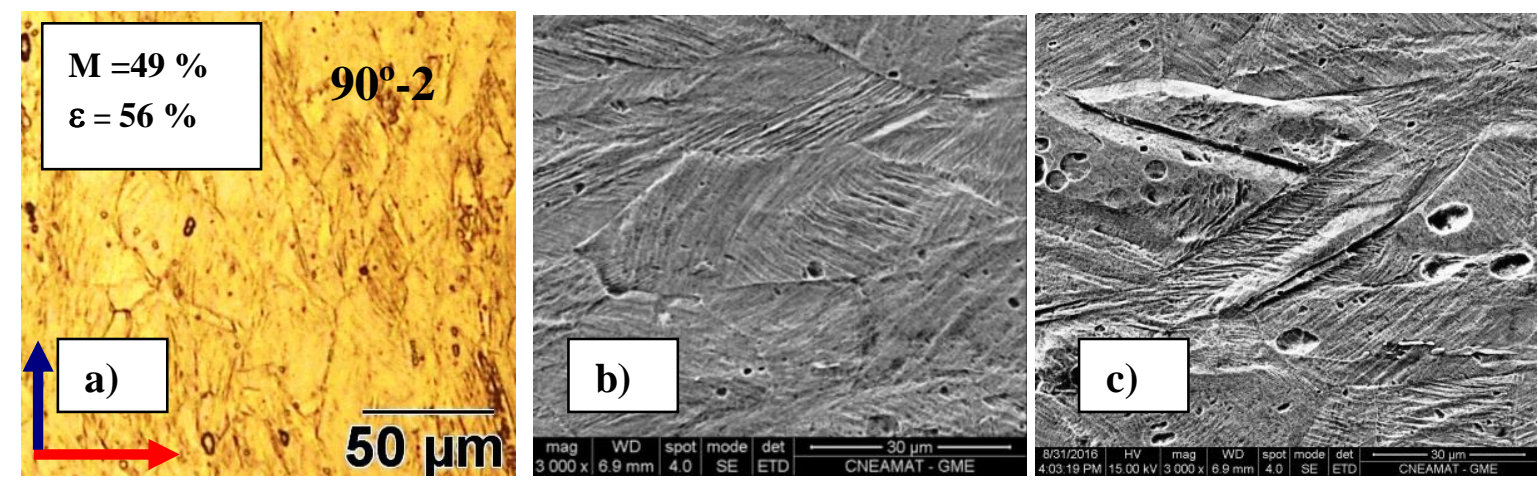

Figura 7: Imágenes: a) microscopia óptica, b) microscopia electrónica de barrido (3000 x) y c) otra zona con amplificación $3000 \mathrm{x}$, para la probeta $90^{\circ}-2$. La flecha roja indica el sentido de laminación y la azul indica el sentido de la deformación.
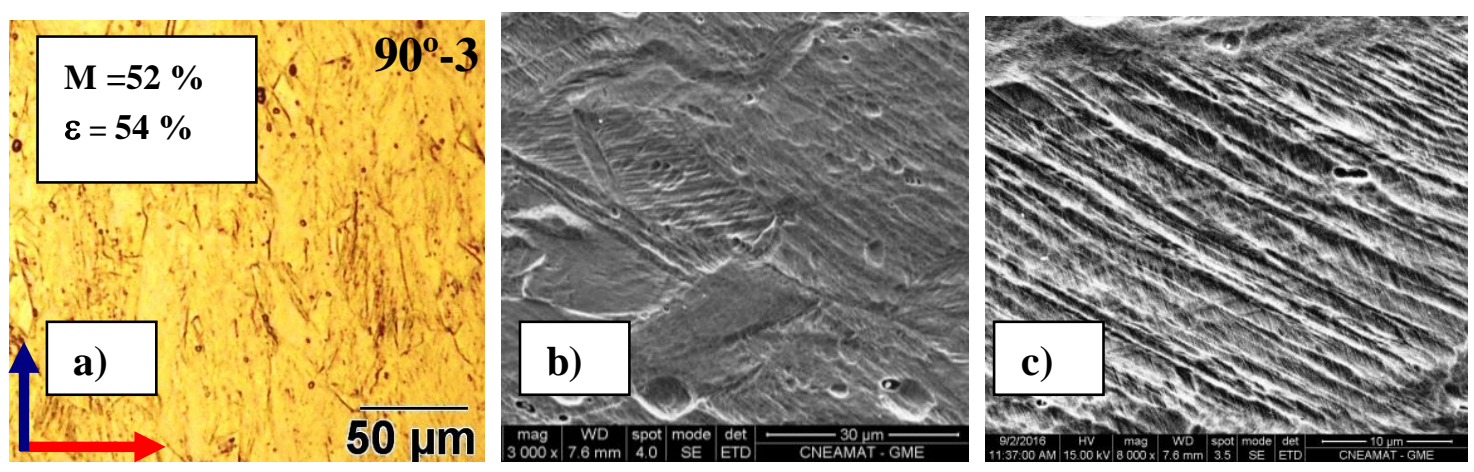

Figura 8: Imágenes: a) microscopia óptica, b) microscopia electrónica de barrido (3000 x) y c) amplificación 8000X, para la probeta $90^{\circ}-3$. La flecha roja indica el sentido de laminación y la azul indica el sentido de la deformación.

De todo el conjunto de probetas ensayadas, se observa que la probeta $0^{\circ}-3$ con deformación plástica final de $60 \%$, sólo tiene un $37 \%$ de martensita. Sin embargo todas las de $90^{\circ}$ con deformaciones parecidas, tienen mayor porcentaje de martensita. Esto se debe probablemente a que la transformación austenitamartensita se origina primero en los bordes de grano (sitios más propicios para su nucleación), y las probetas de $90^{\circ}$ tienen mayor cantidad de bordes de grano en la dirección de deformación [5].

Los resultados en la Tabla 1 indican que el aumento de la deformación plástica está ligado al aumento de la martensita y de la dureza del material. Esto se debe, al incremento de las densidades de defectos (dislocaciones y maclas), a la formación y el crecimiento de la fase martensítica (igual o más dura que la austenítica) y al aumento de las interfaces $[3,5,12]$.

\subsection{Estudio del RMB}

Dado que las señales magnéticas fueron registradas teniendo en cuenta dos ciclos completos de excitación del campo magnético externo, se produjeron cuatro señales de RMB (bucles), dos durante el aumento del campo y dos durante su disminución. Para estudiar las diferencias o similitudes entre ellas, se procedió a hacer un análisis estadístico después de eliminar el ruido.

Se realizó un filtrado de las señales originales mediante el filtro pasabajos de tipo Butterworth con el objeto de mantener una respuesta lo más plana posible en la banda pasante. En la figura 9 se muestran a modo de ejemplo para la probeta $0^{\circ}-3$, los resultados de las señales de RMB obtenidos en su evolución temporal, antes y después del filtrado. Por otra parte, para determinar el grado de semejanza entre los bucles (ver figura 9), se calculó la función RMS ("Root Mean Square”), integrando cada 500 muestras, luego se realizó la correlación cruzada entre ellas. De los resultados alcanzados se observó un alto grado de semejanza entre todos los bucles, no siendo posible distinguir diferencias considerables entre los bucles de "aumento" y los de "disminución” del campo magnético de excitación. 

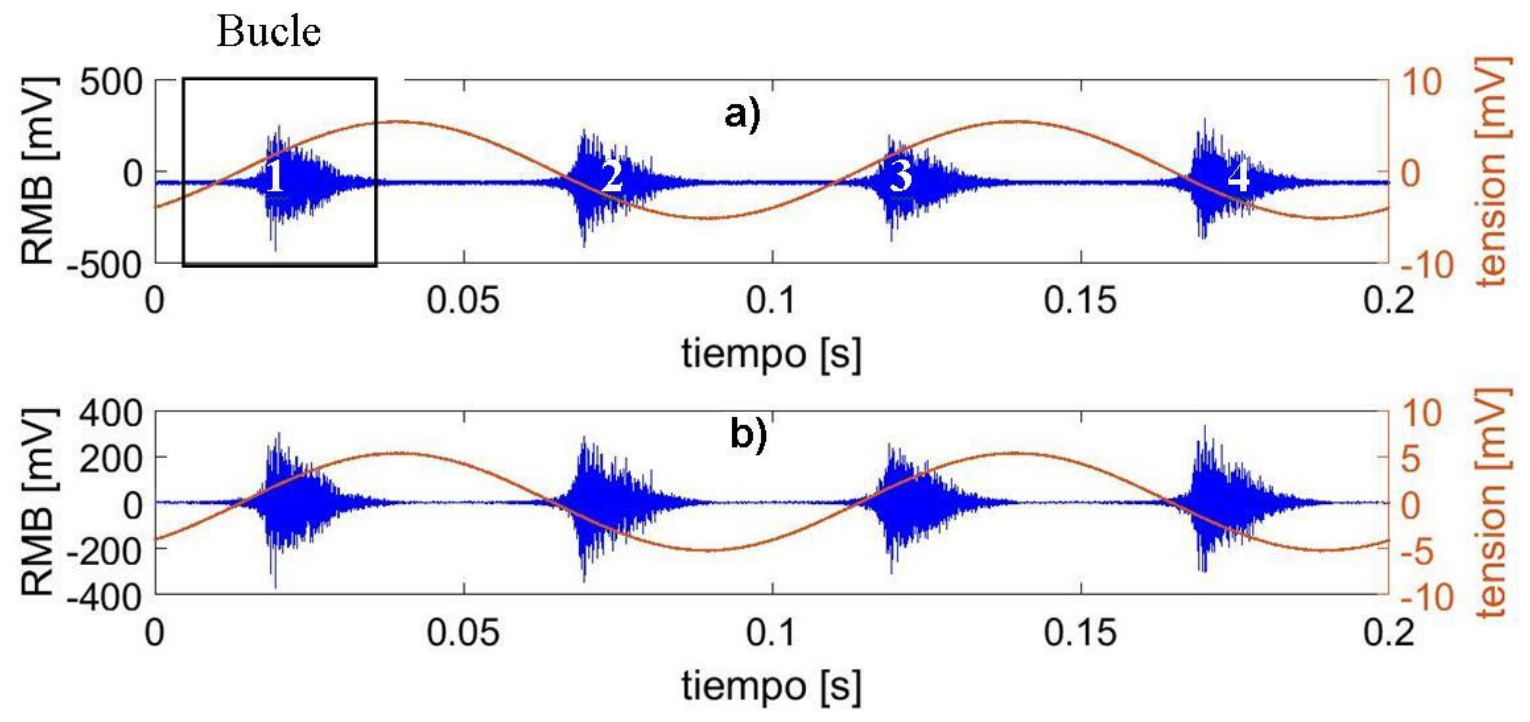

Figura 9: Se muestra la señal de RMB de la probeta $0^{\circ}-3$ a) sin filtrar y b) filtrada.

Si bien los parámetros kurtosis (apuntamiento), asimetría y valor máximo, surgieron en principio para analizar funciones de distribución, su cálculo se puede aplicar a otros casos, permitiendo un más sencillo análisis de las señales. Con la función RMS se construyó una función positiva y más suavizada de cada bucle, a la cual se le calcularon dichos parámetros. Luego se evaluó la energía y el valor RMS de cada bucle en función de la carga aplicada. En la figura 10, a modo de ejemplo, se muestra la kurtosis de cada uno de los bucles del RMB para las probetas a) $0^{\circ}-3$ y b) $90^{\circ}-3$ en función de la carga aplicada. Para la probeta $0^{\circ}-3$, con un $60 \%$ de deformación final, se observa que al comienzo hay fluctuaciones en la kurtosis hasta una carga de $3500 \mathrm{~N}$, luego disminuye y es prácticamente constante hasta $6000 \mathrm{~N}$, aumentando a continuación hasta 7000 $\mathrm{N}$ y luego disminuyendo casi sin dispersión entre los valores de cada bucle. Para la probeta $90^{\circ}-3$, con un 54 $\%$ de deformación final, se reproduce el mismo comportamiento anterior, pero la primera zona de disminución se da entre 3000 N y 5000 N. También se ve que en la última parte del proceso hay menor dispersión de datos.
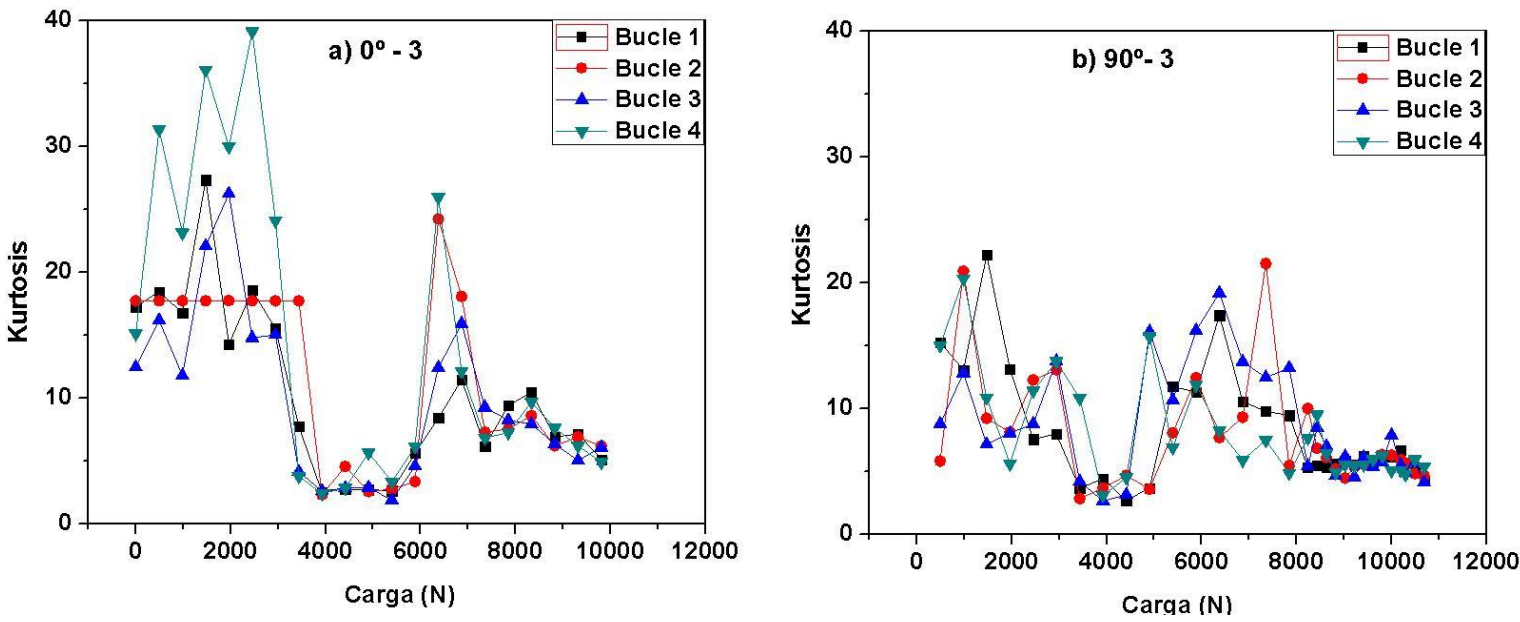

Figura 10: Kurtosis de cada bucle del RMB en función de la carga aplicada, a) probeta $0^{\circ}-3$; b) probeta $90^{\circ}-3$.

En la Figura 11 se muestra la asimetría de los bucles para las mismas probetas, en función de la carga aplicada. Se ve que para la probeta $0^{\circ}-3$ hay mayores fluctuaciones al comienzo y durante el proceso, se produce una disminución entre $3000 \mathrm{~N}$ y $5500 \mathrm{~N}$, luego aumentan los valores y posteriormente disminuyen, pero con menor dispersión de datos. En la probeta $90^{\circ}-3$ se observa prácticamente el mismo comportamiento. 

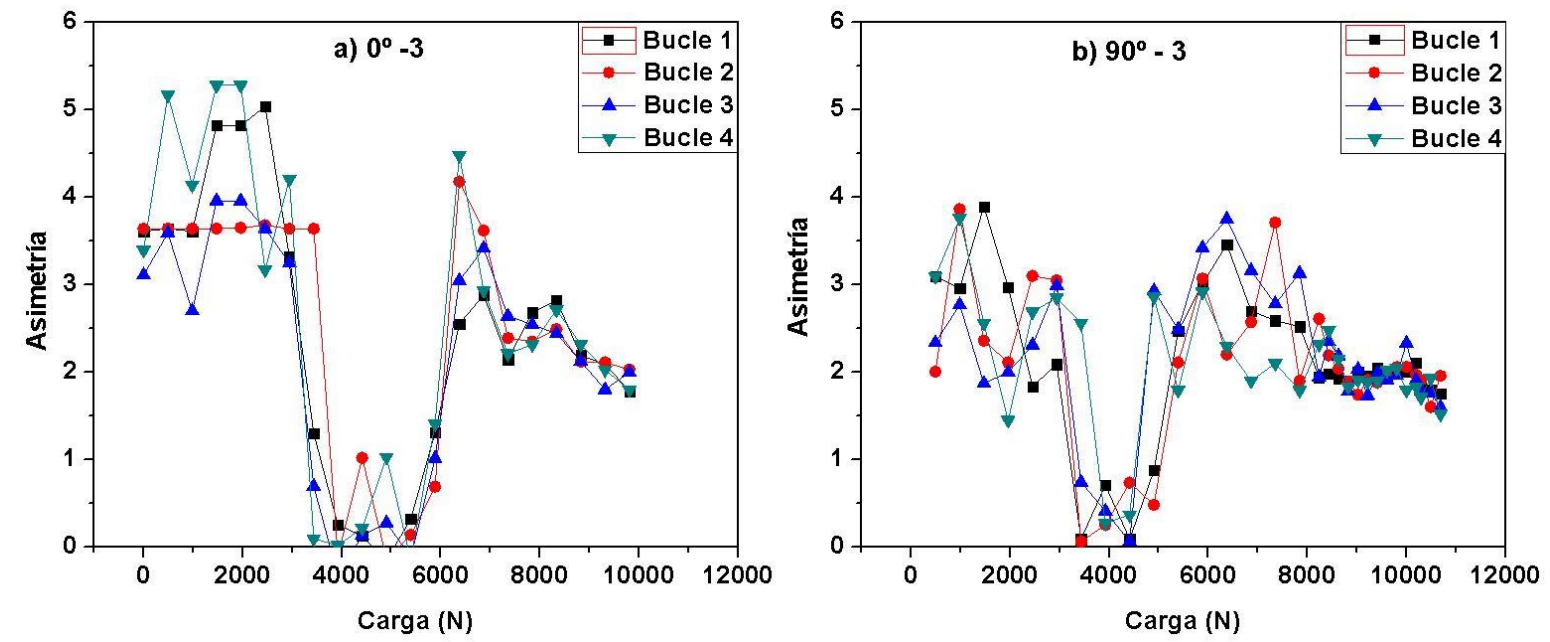

Figura 11: Asimetría de cada bucle del RMB en función de la carga aplicada, a) probeta $0^{\circ}-3$; b) probeta $90^{\circ}-3$.

Si se comparan las figuras 10 y 11, se ve que en ambos casos para la kurtosis y la asimetría, se produce una disminución de sus valores en la zona central de la carga y luego un aumento. En la literatura se encontró, para otros materiales ferromagnéticos, que dichos parámetros aumentan con la deformación [11,12], sin embargo para este material austenítico al producirse una transformación de fase durante el proceso de deformación se obtuvieron otros resultados, dificultándose su interpretación.

En la Figura 12 se muestra el valor máximo de amplitud de cada bucle para las probetas a) $0^{\circ}-3$ y b) $90^{\circ}-3$ en función de la carga aplicada. Se observa que en ambas probetas aumenta el RMB al aumentar la carga, siendo estos valores muchos menores para la probeta $0^{\circ}-3$ en comparación con la probeta $90^{\circ}-3$.
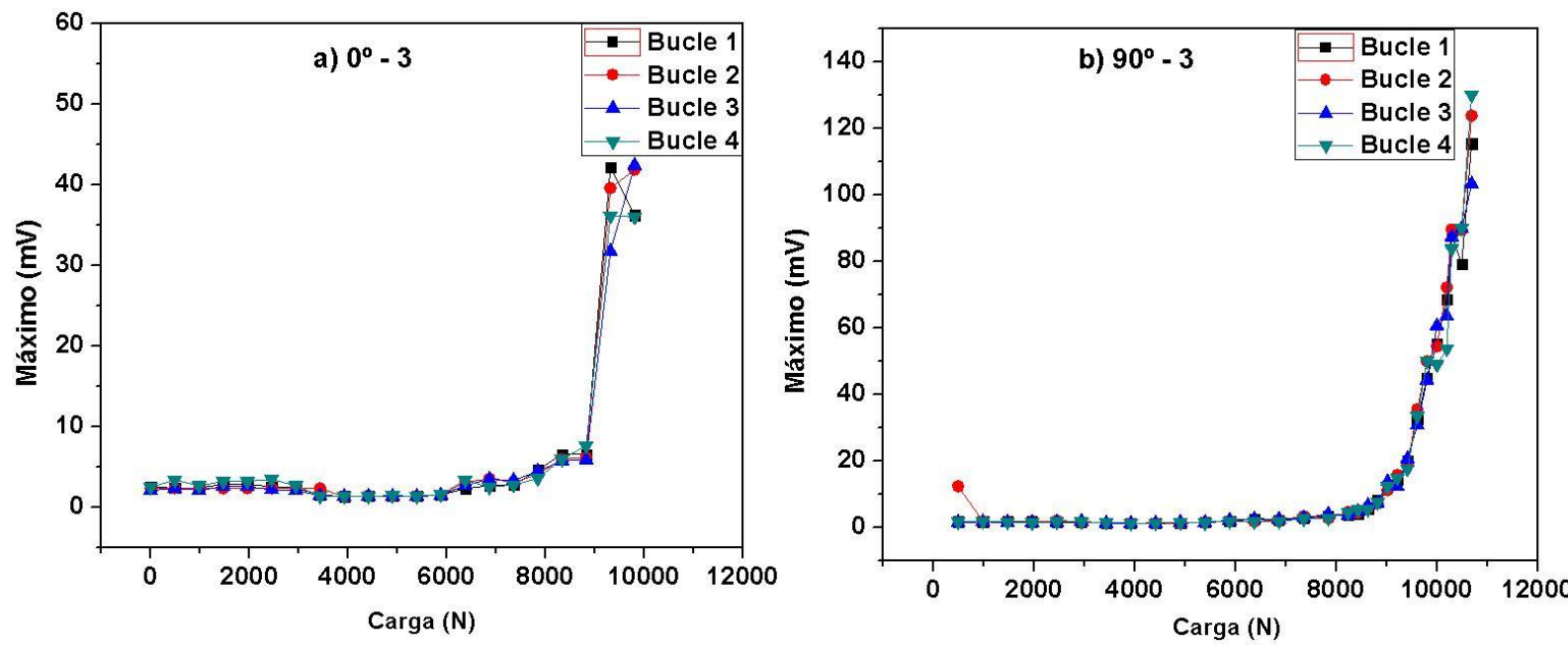

Figura 12: Máximo de cada bucle del RMB en función de la carga aplicada, a) probeta $0^{\circ}-3$; b) probeta $90^{\circ}-3$.

En la Figura 13 se muestra el valor RMS de los bucles para las probetas a) $0^{\circ}-3$ y b) $90^{\circ}-3$ en función de la carga aplicada. Se observa también en este caso que para la probeta $0^{\circ}-3$, los valores de RMS son menores en comparación con los de la probeta $90^{\circ}-3$. 

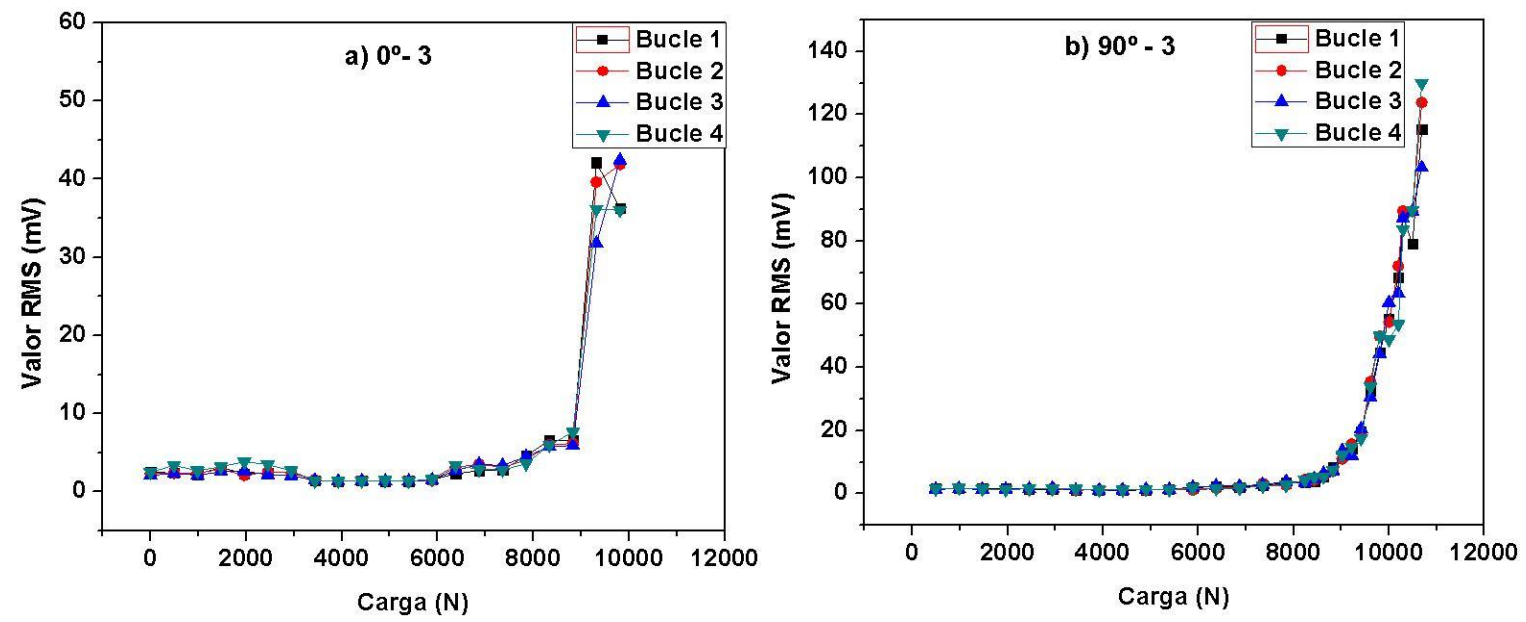

Figura 13: RMS de cada bucle del RMB en función de la carga aplicada, a) probeta $0^{\circ}-3$; b) probeta $90^{\circ}-3$.

En la Figura 14 se graficó la energía de cada bucle, en función de la carga aplicada. Para calcular la energía se empleó la siguiente aproximación:

$$
E_{x}=\frac{1}{Z} \int_{-\infty}^{\infty}|V(t)|^{2} d t \cong \frac{1}{Z} \sum_{n=0}^{N-1}|V[n \Delta t]|^{2} \Delta t
$$

donde $\Delta \mathrm{t}$ es el intervalo temporal (s) entre muestras, $\mathrm{V}(\mathrm{t})$ es la señal muestreada $(\mathrm{mV})$, $\mathrm{Z}$ es la impedancia de la bobina sensora $(\Omega)$ y el subíndice $\mathrm{x}$ representa cada bucle.

En las Figura 14 se ve una casi nula dispersión en los datos correspondientes a cada bucle que se mantiene muy bajos hasta prácticamente los $9000 \mathrm{~N}$ y una energía 10 veces mayos para la probeta la $90^{\circ}-3$.
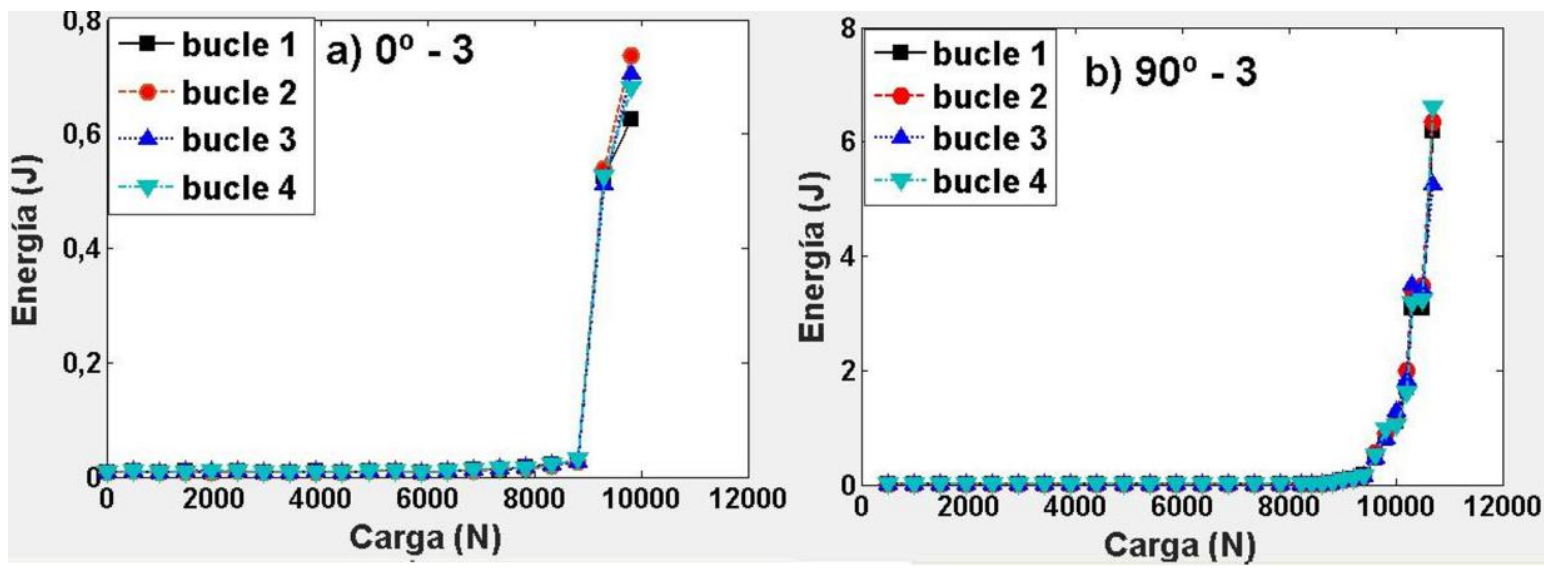

Figura 14: Energía de cada bucle del RMB en función de la carga aplicada, a) probeta $0^{\circ}-3$; b) probeta $90^{\circ}-3$.

Es de notar que por corresponder a magnitudes más globales, los valores de RMS y la energía presentan menor dispersión en sus valores.

En todos los parámetros estudiados, en general se verificó la presencia de mayores valores para la probeta $90^{\circ}-3$ comparados con los de la $0^{\circ}-3$. En este último caso, la mayoría de los granos están alargados en el sentido de laminación que coincide con el de la tracción axial, pero en la $90^{\circ}-3$, los granos se encuentran alargados en la dirección perpendicular al sentido de la tracción. Por lo tanto al aumentar la carga, la probeta $90^{\circ}$-3 tiene mayor cantidad de obstáculos (bordes de grano) en la dirección de deformación, produciendo mayor RMB $[8,11]$. 
En la Figura 15 se muestran los valores de RMS y de energía, obtenidos al final del proceso de deformación hasta rotura, en función de la martensita final. Cada punto corresponde a una de las probetas ensayadas. Se observa que aumenta el valor de RMS y la energía de las señales del RMB, con el aumento de la martensita, estos resultados experimentales se corroboran en la literatura [3, 4, 14-17]. Allí se explica que las interfaces austenita-martensita puede ser consideradas como fuertes puntos de anclaje. Además se ha sugerido que los bordes de grano son sitios densos de fijación y liberación repentina de paredes de dominios, que generan un gran pico en el perfil del RMB [15]. Por tales motivos se puede explicar que las probetas deformadas en sentido perpendicular al sentido de laminación, tengan mayores porcentajes de martensita ya que contienen mayor cantidad de obstáculos (bordes de grano) en la dirección de la deformación. A medida que aumenta la deformación, crece la fase martensita generando más defectos que actúan también como obstáculos, produciendo un aumento en los valores del RMS y la energía.

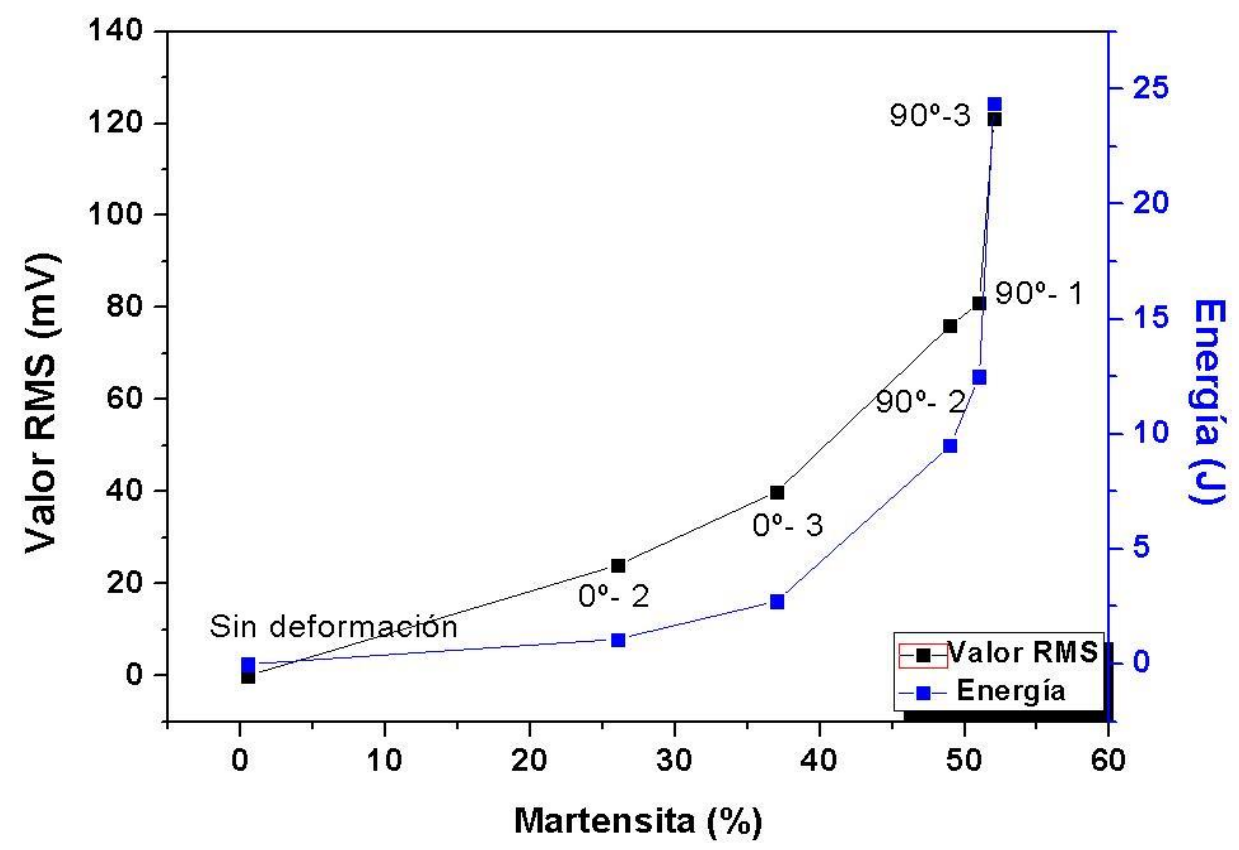

Figura 15: Valores finales de RMS y energía, en función del porcentaje final de martensita.

\section{CONCLUSIONES}

En este trabajo se continuó el estudio de los ensayos de tracción uniaxial hasta rotura en probetas del acero AISI 304, comprobándose mediante el RMB la creación de la fase martensita. Las probetas fueron caracterizadas y estudiadas metalográficamente, comprobándose el aumento de las maclas y de la cantidad de agujas de martensita con la creciente deformación.

Mediante el análisis de la correlación de señales, se concluyó la equivalencia de la función RMS de los cuatro bucles producidos durante los dos ciclos de excitación magnética.

El particular comportamiento de los parámetros de kurtosis y asimetría de los bucles, debe ser analizado posteriormente con mayor profundidad.

Los valores máximos de amplitud, RMS y energía, resultaron mayores en los ensayos de las probetas cortadas perpendicularmente a la dirección de laminación de la placa.

Se comprobó que con la mayor deformación aumentaba la fase martensita (magnética) y se generaba mayor cantidad de defectos que actuaban como obstáculos al movimiento de las paredes de los dominios magnéticos, generando así un aumento en la energía necesaria para poder superarlos.

\section{AGRADECIMIENTOS}

A Silvia Adriana Domínguez por el servicio del microscopio electrónico de barrido, Grupo de Microscopía Electrónica, Gerencia Materiales, Comisión Nacional de Energía Atómica, Buenos Aires, Argentina.

A la División Metalografía y Ensayos Termomecánicos, Gerencia Materiales, Comisión Nacional de Energía Atómica, Buenos Aires, Argentina. 


\section{BIBLIOGRAFÍA}

[1] SMITH ,W. F., Structure and Properties of Engineering Alloys, 1993.

[2] MOORTHY V., VAIDYANATHAN S., JAYAKUMAR T., et al., "On the influence of tempered microstructures on magnetic Barkhausen emission in ferritic steels", Philosophical Magazine A, v. 77, pp. 14991514, 1998.

[3] AHMADZADE BEIRAKI, E., MAZINANI, M., KASHEFI, M., "Examination of Barkhausen noise parameters for characterisation of strain-induced martensitic transformation in AISI 304 stainless steel", Insight, v. 58, n. 6, pp.297-301, June 2016.

[4] JARRAHI, F., KASHEFI, M., AHMADZADE-BEIRAKI, E. "An investigation into the applicability of the Barkhausen noise technique in the evaluation of machining properties of high carbon steel parts with different degrees of spheroidisation", Journal of Magnetism and Magnetic Materials, v. 385, pp. 107-111, 2015.

[5] EASTERLING, K., E., LEN, I. I., "The Nucleation of Martensite in Steel”, Acta Metallurgica, v. 24, pp. 334-341, 1971.

[6] STEFANITA, C. G., From Bulk to Nano. The Many Sides of Magnetism, Springer Series in Materials Science, 2008.

[7] JILES, D., Introduction to Magnetism and Magnetic Materials, Chapman and Hall, 1995.

[8] ROCÍO, N.A.M., NICOLAS, M. N., RUZZANTE, J., et al., "Correlation between Martensitic Phase Transformation and Magnetic Barkhausen Noise AISI 304 Steel", Procedia Materials Science, v. 9, pp. 435443, 2015.

[9] MORAIS, V. L., "Estudo Comparativo da Deformação a Frio e da Resistência À Corrosão Nos Aços Inoxidáveis Austeníticos AISI 201 e AISI 304", Dissertação de M.Sc em Engenharia, Universidade de São Paulo, SP, Brasil, 2010.

[10] MILAD, M., ZREIBA, N., ELHALOUANI, F., et al., "The effect of cold work on structure and properties of AISI 304 stainless steel”, Journal of Materials Processing Technology, v. 203, p. 80-85, 2008.

[11] ROCÍO, N.A.M., NÚÑEZ, N., LÓPEZ, I. M. P, et al., "Analisis de Ruido Magnetico Barkhausen en acero AISI 304", In: $28^{\text {th }}$ Internacional Congreso of Condition Monitoring and Diagnostic Engineering, $\mathrm{X}$ Congreso Regional de Ensayos No Destructivos y Estructurales, ISBN: 978-987-23957-6, 1-4 de diciembre 2015.

[12] AMITAVA MITRA, P. K., SRIVASTAVA, P.K., BHATTACHARYA, D.K. "Ferromagnetic Properties of Deformation-Induced Martensite Transformation in AISI 304 Strain", Metallurgical and Materials Transactions A, V. 35A, February 2004.

[13] DING, S., TIAN, G.Y., MOOORTHY, V., et al., "New feature extraction for applied stress detection on ferromagnetic material using magnetic Barkhausen noise", Measurement, v. 73, pp. 515-519, 2015.

[14] DING, S. , TIAN, G-Y., DOBMANN, G., et al., "Analysis of domain wall dynamics based on skewness of magnetic Barkhausen noise for applied stress determination", Journal of Magnetism and Magnetic Materials, v. 421, pp. 225-229, January 2017.

[15] HAUSILD P., KOLARÍK K., KARLÍK M., "Characterization of strain-induced martensitic transformation in A301 stainless steel by Barkhausen noise measurement", Material \& Design, v.44, pp. 548-554, 2013.

[16] LEBEDEV A. A., KOSRCHUCK V. V., "Influence of phase transformations on the mechanical properties of austenitic stainless steels", International Journal of Plasticity, v. 16, pp. 749-767, 2000.

[17] DAS, A., TARAFDER, S., CHAKRABORTI, P.C. "Estimation of deformation induced martensite in austenitic stainless steels", Materials Science and Engineering A, v. 529, pp. 9-20, August 2011. 